\title{
MOLECULAR AUTORADIOGRAPHY:
}

\section{THE $\beta$-RAY COUNTING FROM SINGLE VIRUS PARTICLES AND DNA MOLECULES IN NUCLEAR EMULSIONS*}

\author{
CYRUS LEVINTHAL AND CHARLES A. THOMAS, Jr. * \\ Physics Department, University of Michigan, Ann Arbor, Mich. (U.S.A.)
}

\section{INTRODUCTION}

At present two methods are in general use for the detection and measurement of radioactivity. Particle counters can be used if the level of activity is not low compared to the background count from cosmic rays and natural radioactivity, and autoradiographic methods can be employed to measure the amount and localization of label. There is, however, an important class of problems for which these methods have not been very satisfactory. The work of $\mathrm{LEvi}^{1}$ and that of FICQ et al. ${ }^{2}$ with track autoradiographs demonstrate that it is possible to measure the activity in small cells which are visible in the microscope; however, the measurement of the amount of label in individual submicroscopic particles has not been accomplished.

If the source of radioactivity is sufficiently small, as in the case of viruses and most bacteria, the objective of counting the emissions from single particles can be realized by the use of an electron-sensitive photographic emulsion. If a particle containing a $\beta$-emitting isotope is imbedded in the nuclear emulsion, and the exposure time is adjusted so that several disintegrations occur before development, then one can observe a number of electron tracks, each consisting of rows of silver grains, emanating from a small region. These groups of tracks are called "stars" (see Fig. I).

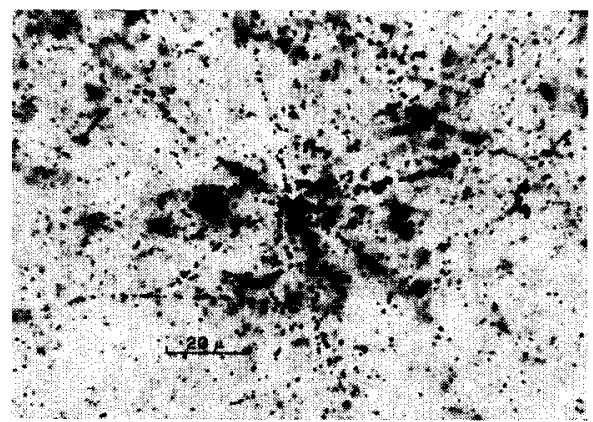

Fig. I. A photomicrograph taken of a star residing on the surface of the emulsion with a $90 \times$ oil immersion objective with a numerical aperture of $\mathbf{I} .32$. Because of the small depth of field only a few of the many tracks from this particle are seen in the photograph.

* This research was supported by grant RG 4557 from the U.S. Public Health Service and by grant I 8 from the Michigan Memorial Phoenix Project.

National Research Council Fellow in Medical Sciences for which funds were provided by the Rockefeller Foundation.

References $p .465$. 
Since the emitting region will be less than a few microns, tracks from stray radiation and cosmic rays are scarcely ever confused with an emission from the particle itself. Thus the problem of "background" is much reduced by the spatial coincidence of the emissions from the particle itself.

${ }^{32} \mathrm{P}$ has a particularly convenient half-life of $\mathrm{I} 4.3$ days and a high maximum beta energy which makes counting relatively simple. Furthermore, ${ }^{32} \mathrm{P}$ is the only isotope which can be used for labeling nucleic acids at a sufficiently high level. The method to be reported here has been applied to DNA molecules, virus particles, and bacteria labeled with ${ }^{32} \mathrm{P}$, and the method is sufficiently sensitive to measure the number of disintegrations from a single particle if that number is as high as ro- 15 per month. The growth processes which we have been studying with bacteriophage require only a few hours for their completion. It is therefore possible to finish the biological parts of the experiments before too many ${ }^{32} \mathrm{P}$ disintegrations have occurred to disturb the normal growth mechanisms, and still have a sufficiently high level of labeling for the detection of stars in the emulsion after reasonable exposure times.

The average phosphorus content of bacteriophage particles has been repeatedly determined and shown to be independent of the conditions of growth ${ }^{3}$. The number of ${ }^{32} \mathrm{P}$ atoms in a single particle can be determined from the total phosphorus content of the particle if the specific activity of the growth medium is known. Thus these phage are ideal test particles for establishing the efficiency of the emulsion to register observable tracks.

The molecular weight of star-forming particles grown in a uniformly labeled medium can be calculated from the number of stars, if the mass of material in starforming particles is known, or from the average number of rays per star if the chemical composition of the star-forming particle is known.

This paper will describe the details of the use of the emulsion and the results obtained with $\mathrm{T}_{2}$ and $\mathrm{T}_{4}$ bacteriophage and the DNA extracted from these viruses ${ }^{4,5}$.

\section{EXPERIMENTAL}

The photographic methods will be described in considerable detail. However, this does not imply that the procedures we have developed are necessarily optimal, but only that they work. Several of the steps have been studied in detail, and a number of difficulties have been experienced. There are also many points of procedure which we have held constant only because they were included in the first successful attempt. An effort will be made in this description to distinguish between those parts of the procedure which are known to be important, and those which have not been investigated.

\section{Plate construction}

The Ilford G-5 emulsion (Ilford Limited, Ilford. London) which is stored at $5^{\circ} \mathrm{C}$ in a shielded box described below is melted in a glass container in a waterbath at $50^{\circ} \mathrm{C}$. Care is taken to avoid touching the emulsion with any material except glass. However, the effect of stainless steel implements has not been tested. The liquid emulsion is drawn into a io ml syringe (without the metal needle) the plunger of which has been fitted with a ratchet device which clicks audibly at $1 / 2 \mathrm{ml}$ intervals. The solution containing the radioactive particles is drawn into the syringe along with the emulsion, and one $\mathrm{ml}$ of air is then taken in to facilitate the mixing of the solution in the syringe. The emulsion and the sample solution are mixed approximately $3: \mathrm{I}$. The emulsion mixture can now be deposited on $I^{\prime \prime} \times 3^{\prime \prime}$ microscope slides which have been coated with a thin layer of gelatin to References $p .465$. 
improve the adherence of the emulsion to the surface. (These slides are supplied by Ilford, Ltd.) The slides are previously prepared by inscribing a serial number across one end and cementing two or three glass rings of $1.5 \mathrm{~cm}$ in diameter with pure paraffin. The plates are warmed to about $40^{\circ} \mathrm{C}$ on a level brass plate which has copper coils on its lower surface, through which either warm or cold water can be circulated. The emulsion mixture is deposited in the rings to form a wet layer approximately $2.5 \mathrm{~mm}$ thick. The brass plate is then chilled to about $15^{\circ} \mathrm{C}$ (it is important not to reduce the temperature below the dewpoint of the room) and the emulsion gels. A sharp needle is used to separate the emulsion from the rings, and the rings are then removed. It is important to remove any air bubbles which might be formed between the emulsion and the glass, since they will expand at the time of development. This can be done by inverting the plate and rolling a needle between emulsion and the glass to lift the emulsion layer free from the glass, and then allowing it to fall back down into contact. When the emulsion layer has been properly prepared, the plates are dried. These operations are performed at least $2 \mathrm{ft}$ from a 40 watt bulb covered by a Wratten No. 2 safelight filter.

\section{Drying plates}

Since wet emulsions are not sensitive to beta-rays, exposure does not begin until emulsions have lost most of their moisture. It is desirable to know roughly the time at which exposure begins. Therefore, the slides bearing the gelled emulsions are transferred to a light-tight box containing baffied shelves, a gentle centrifugal fan and open dishes of silica gel, where they are rapidly dried. Under these conditions at room temperature, the emulsions will lose $50 \%$ of their moisture in 2.3 hours and $90 \%$ in 7.2 hours. For long exposures, a small uncertainty is not important; however, this uncertainty gains importance with shorter exposures. As a matter of practice, exposure was assumed to begin three hours after the emulsions begin to dry. After about I 6 hours of drying, the plates are transferred to boxes for storage.

\section{Storage}

The plates are stored at $5^{\circ} \mathrm{C}$ under $1 / 2$ inch of lead, 3 inches of steel, and $1 / 4$ inch of copper. This shield, which is in the form of a box, eliminates all of the cosmic ray background except the fast mesons which cannot be confused with the electron tracks from ${ }^{32} \mathrm{P}$. The plates are kept in lighttight boxes, through which a stream of humidified nitrogen passes. Dry nitrogen is humidified by passing the gas over a saturated solution of $\mathrm{CaCl}_{2} \cdot 6 \mathrm{H}_{2} \mathrm{O}$ at $5^{\circ} \mathrm{C}$. If equilibrium were attained, this solution would provide an R.H. of about $40 \%$. If the gas is too dry, there is difficulty with the emulsion peeling off the glass. Under these conditions of storage there is a negligible amount of latent image fading; however, it has not been established that the nitrogen atmosphere is necessary in order to eliminate latent image fading.

\section{Developing procedure}

An important feature of the developing procedure is the removal of plates from the storage shield. It was found that if plates were repeatedly warmed to room temperature and recooled, the latent image faded. Thus, in a series of plates to be developed sequentially, which are all stored in the same box, the removal of the early members of the series will result in the fading of the later members. This has been overcome by placing all those plates to be developed at a single time in the same box, or by employing special insulated boxes which allow the withdrawal of a single plate without those remaining warming or receiving surface condensation from the atmosphere.

The developing procedure used here is somewhat less complicated than that which is usually used for thick emulsions by workers in the field of nuclear physics ${ }^{6}$. In their case, it is necessary to avoid any distortion of the emulsion which might change the curvature of the tracks. Since we are dealing only with electron tracks which scatter heavily in any case, distortion is not significant.

The following solutions are used in the developing process. Developer: $\mathrm{H}_{2} \mathrm{O}_{3} 1, \mathrm{Na}_{2} \mathrm{SO}_{3} 54 \mathrm{~g}$, Amidol $13.5 \mathrm{~g}, \mathrm{I} \% \% \mathrm{KBr}$ solution $24 \mathrm{ml}$. Fix: $\mathrm{H}_{2} \mathrm{O}_{3} \mathrm{l}, \mathrm{Hypo}$ I $200 \mathrm{~g}, \mathrm{NH}_{4} \mathrm{Cl} 2 \mathrm{I} \mathrm{g}, \mathrm{NaHSO}_{3} 2 \mathrm{I} \mathrm{g}$. Acid stop: $\mathrm{H}_{2} \mathrm{O}_{3} \mathrm{l}$, glacial acetic acid $30 \mathrm{ml}$.

The fix and acid stop are stored, but it is important that the developer be made up fresh on every occasion.

The plates are plunged into $5^{\circ} \mathrm{C}$ developer and soaked for 50 minutes. The rack containing the plates is then transferred to a $\mathrm{I}: 2$ dilution of the same developer adjusted to $2 \mathrm{I}^{\circ} \mathrm{C}$ where they are developed for 40 minutes. The plates are rinsed and soaked in acid stop at $5^{\circ} \mathrm{C}$ for another 50-60 minutes and then begin a 20 -hour fixation in a large excess of fix. Gentle agitation provided by nitrogen streaming through a glass frit, speeds up the fixing process. The temperature of the fix should not exceed $25^{\circ} \mathrm{C}$. The plates are rubbed gently with a chamois to remove any surface deposit of silver and placed into the wash. The wash water must be very gently flowing and not below $18^{\circ} \mathrm{C}$, otherwise excessive distortion of the emulsion results. Washing proceeds for approximately 4 to 6 hours; the water draining from the plates is tested for the absence of hypo by a colorimetric test ${ }^{\star}$ and the plates set aside to dry slowly in the air. The plates are not exhaustively dried.

\footnotetext{
* In this case Dupont "Defender" wash test was employed.
} 


\section{Counting stars}

The information which can be obtained from any one plate is the number of stars per unit volume of the solution originally mixed with the emulsion, and the distribution of tracks among the particles which form stars. Two different methods have been used to measure the former quantity. First, one can count all of the stars in a known volume of dried emulsion, and from this calculate the number of radioactive particles in the original wet emulsion. This method is somewhat unsatisfactory, since it depends on a random distribution of particles through the emulsion; and for the determination of the absolute number of particles it depends on an accurate determination of the shrinkage factor of the emulsion.

A second, and generally more satisfactory method, is the use of indicator particles to determine the volume of solution counted. Two kinds of indicator particles have been used. In the early work $^{5}$ a solution of the radioactive isotope polonium $2 \mathrm{IO}$, which emits alpha particles, was mixed with the solution containing the ${ }^{32} \mathrm{P}$-labeled particles, and the ratio of alpha particles emitted by the polonium to stars was measured in the developed emulsion. These indicator particles had numerous disadvantages and were finally replaced by polystyrene latex spheres of approximately ten microns in diameter. These spheres were prepared by Dr. John VAnderhoff, Physics Department, Dow Chemical Company, Midland, Michigan, and are mixed with the final suspension of radioactive particles. Since the spheres can be easily counted in a bacterial counting chamber, and can also be seen in the developed emulsion, they make ideal indicator particles. In general, the material which is mixed with the emulsion contains approximately ${ }^{4}{ }^{4}$ spheres per ml and in the vicinity of $1 \mathrm{O}^{4}$ radioactive particles per $\mathrm{ml}$.

The plates are examined in a binocular microscope using oil immersion objectives. A $22 \times$ objective with a NA of 0.65 is used for searching and for detailed examination of a given star, a high power oil objective with a $\mathrm{NA}=\mathrm{I} .32$, is used. $10 \times$ oculars are generally used, and these are fitted with square diaphragms to simplify the searching process. In general, the observer must obtain both the ratio of stars to spheres in any arbitrary region of the plate, and the number of rays in randomly selected stars. Counting of the individual tracks from a star is a difficult but not impossible process. There is often some uncertainty of one or two tracks in stars which have a number of rays of the order of from 15 to 20 ; in stars which have about 40 rays the number can only be estimated. Since a fortuitous crossing of two electron tracks might be mistaken for a star of 4 rays, an arbitrary decision has been made not to count a star unless it has 5 or more tracks. This requires a correction for a slight distortion of the observed average star size, which will be explained below. Since the average number of rays per star can be affected by non-random selection of stars, as well as by errors in counting rays in particular stars, it was decided that every star which came within the square field of view should be counted unless it resided within Io microns of the upper or lower surface of the emulsion, and if it was not superimposed on an indicator sphere or a piece of foreign matter.

\section{Growth of labeled phage}

Phage $\mathrm{T}_{2 \mathrm{r}_{1} \mathrm{~h}^{+} \text {were grown }}{ }^{7}$ in bacteria which had been growing in tris-glucose media ${ }^{8}$ supplemented with $10^{-4} \mathrm{~g}$ peptone $/ \mathrm{ml}$ and containing ${ }^{32} \mathrm{P}$ at a specific activity of 100 to $200 \mathrm{mc} / \mathrm{mg}$. After one hour the bacteria were lysed with $N /$ Ioo $\mathrm{KCN}^{9}$ and purified by differential centrifugation. The phage were treated with DNase and in some experiments adsorbed to bacteria to affect further purification before mixing with the emulsion. In certain experiments growth was arrested by the addition of chloramphenicol at $20 \gamma / \mathrm{ml}$. It was established that, under the conditions of adsorption either with chloramphenicol or without, there was no loss of radioactivity from the bacterial pellet during the time required for the emulsion to gel.

\section{STATISTICS}

Since the data derived from counting the number of stars and number of rays per star requires statistical interpretation, it is important to consider in some detail how a random decay of ${ }^{32} \mathrm{P}$ atoms in a population of particles containing ${ }^{32} \mathrm{P}$ will lead to a population of stars.

In the experiments to be discussed here, uniform labeling was obtained by prolonged growth of the bacteria and virus particles in a medium containing a fixed ratio of ${ }^{32} \mathrm{P}$ and ${ }^{31} \mathrm{P}$. This type of growth is assumed to lead to random uniform labeling of the phosphorus atoms with ${ }^{32} \mathrm{P}$. We will designate the probability that any phosphorus atom has emitted a $\beta$-particle as $\beta$. This is the product of two probabilities: the probability that the particular atom is labeled, and the probability that a particular ${ }^{32} \mathrm{P}$ atom has decayed. The latter probability will be a function of time.

\section{Uniform population}

If each potential star-former has an identical number of phosphorus atoms, and if this number is

References $p .465$. 
large in comparison to the number of disintegrations which take place, then the disintegrations will be distributed over all of the star-formers in a Poisson distribution, given by

$$
P(\bar{r}, r)=\frac{\mathrm{e}^{-\bar{\gamma} \bar{\gamma}^{r}}}{\mathrm{r} !}
$$

where $P(\bar{r}, r)$ is equal to the fraction of stars which have $r$ rays when there are on the average $\bar{r}$ rays per star. It was shown by Hershey, Kamen, Kennedy and Gest ${ }^{3}$ that T2 phage grown under a variety of conditions had the same average phosphorus content and that essentially all of the phage particles in a labeled population had the same probability of being inactivated by the decay of incorporated ${ }^{32} \mathrm{P}$. Thus it seems very reasonable to assume that every phage particle has the same phosphorus content. If each particle contains exactly $t$ phosphorus atoms, the mean number of disintegrations per particle would be $\beta t$. The variance, $\sigma^{2}$, about this mean should be equal to the mean itself for a Poisson distribution. The average number of rays per star as observed in a population will be designated $\bar{r}_{\text {obs }}$. The number of rays from a particular star will be designated $r_{i}$ and $n$ will be the total number of stars in a given population. The experimentally determined quantity which will be used to estimate $\sigma^{2}$ is $s^{2}$ defined as

$$
s^{2}=\frac{\sum_{i=\mathrm{I}}^{i=n}\left(\bar{r}_{\mathrm{obs}}-v_{i}\right)^{2}}{n-\mathrm{I}}
$$

However, stars of less than 5 rays are not counted or recorded. Thus the observed average $\bar{r}_{\text {obs }}$ can never fall below 5. To correct for the fact that stars with a small number of rays would not be counted, we employ the following expressions which relate $\bar{\gamma}_{\text {obs }}$ and the expected variance with the true quantities which would have been expected if an exact Poisson distribution were involved and all stars were counted.

$$
\bar{r}_{\mathrm{obs}}=\frac{\bar{r}-\sum_{0}^{4} r P(r)}{\sum_{5}^{\infty} P(r)}
$$

$$
\begin{gathered}
\sigma_{\text {obs }}^{2}=\bar{r}_{\text {obs }}-\bar{r}^{2}{ }_{\mathrm{obs}} \\
\text { where } \bar{r}^{2}{ }_{\mathrm{obs}}^{\infty}=\frac{\sum_{5}^{2} r^{2} P(r)}{\sum_{5}^{\infty} P(r)}
\end{gathered}
$$

These quantities have been calculated and are shown in Fig. 2. If one can assume star-formers which contain identical numbers of phosphorus atoms, then one can correct the observed mean for uncounted stars of size less than 5 by reading off the value of $v$ corresponding to the observed

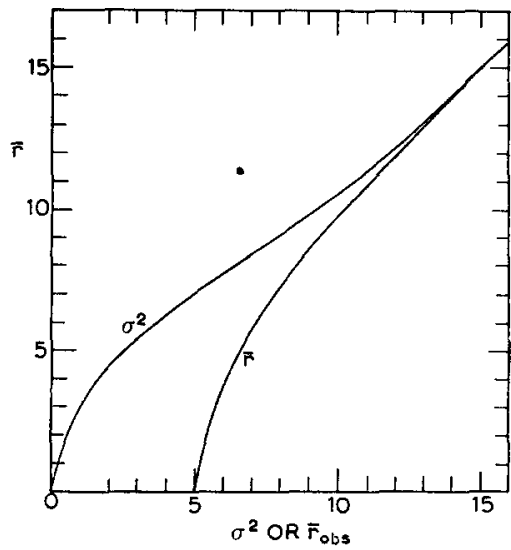

Fig. 2

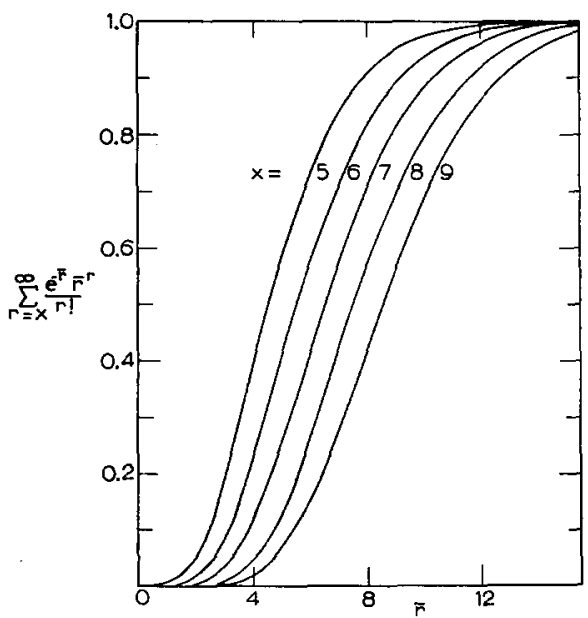

Fig. 3

Fig.2. Distortion of observed mean and variance resulting from exciusion of stars with less than five rays.

Fig. 3. The fraction of stars with 5 rays (6 rays, etc.) or more as a function of the average number of rays per star.

References p. 465 . 
value of $\bar{v}_{\text {obs. }}$ The expected valuc of $\sigma^{2}$ is smaller than the mean when the mean is small, and can be found by reading off the abscissa corresponding to the true mean value evaluated from $\bar{r}_{\text {ois }}$.

The number of stars of all sizes also depends on the average number of rays per star, $\bar{r}$. In Fig. 3 the fraction of stars manifested as stars of 5 or greater, or 6 and greater, etc., is plotted as a function of the mean number of rays per star, again assuming a monodisperse population of star-formers.

\section{Non-uniform population}

Since in general one does not expect all of the star-formers to have exactly $t$ phosphorus atoms, we now ask what kind of population of star sizes one expects from a collection of star-formers with different numbers of phosphorus atoms. If $S(r)$ is the number of stars with exactly $r$ and $N(t)$ is the number of molecules with exactly $t$ phosphorus atoms we have:

$$
S(r)=\sum_{t=\mathrm{I}}^{\infty} N(t) P(\beta t, \boldsymbol{r})
$$

the sum representing the contribution of all different molecules to that class of stars of size $r$. $P(\beta t, \gamma)$ is the Poisson distribution $\mathrm{e}^{-\beta t}(\beta t) r / \gamma !$. The average number of rays per star, neglecting the effect of uncountable stars of size less than five, becomes

$$
\vec{r}-\frac{\sum_{r=0}^{\infty} \sum_{t=\mathrm{I}}^{\infty} N(t) r P(\beta t . r)}{\sum_{r=0}^{\infty} \sum_{t=\mathrm{I}}^{\infty} N(t) P(\beta t, r)}
$$

Interchanging the order of summation we have:

$$
\bar{r}=\frac{\beta \sum_{t=1}^{\infty} t N(t)}{\sum_{t=1}^{\infty} N(t)}=\beta t_{n}
$$

where $t_{n}$ is the number average number of phosphorus atoms per molecule.

In a similar fashion it is possible to calculate the variance for the population of stars. This is found to be:

$$
\begin{gathered}
\sigma_{r}^{2}=\beta^{2} t_{n}{ }^{2}\left(t_{w} / t_{n}-\mathbf{I}\right)+\beta t_{n} \\
\text { or } \sigma_{r}^{2}=\left[\frac{\sigma_{i}}{t_{n}}\right]^{2} \cdot \bar{r}^{2}+\bar{r}
\end{gathered}
$$

where $t_{?}$ is the weight average number of phosphorus atoms per molecule defined:

$$
t_{w}=\frac{\sum_{I}^{\infty} t^{2} N(t)}{\sum_{I}^{\infty} t N(t)}
$$

and $\sigma^{2}$ is defined as:

$$
\sigma^{2}{ }_{t}=\frac{\sum_{\mathrm{I}}^{\infty}\left(t-t_{n}\right)^{2} N(t)}{\sum_{\mathrm{I}}^{\infty} N(t)}
$$

The distribution of star sizes can be found explicitly from equation 5 by substituting any assumed distribution for $N(t)$. One of the most important distributions, namely that resulting from the random polymerization of monomer units, or the random degradation of a linear polymer molecule is characterized by a ratio of weight to number average degree of polymerization of $2^{12}$. In this

References p. 465 . 
case we see from equation 8 that $\sigma_{r}^{2}=\bar{r}^{2}+\bar{r}$. If, for instance, the observed ${ }^{r}$ were Io, this would result in the large value of I Io for $\sigma_{r}{ }^{2}$. Thus it would be very simple to distinguish a homogeneous population of star-formers from a moderately polydisperse population.

The standard deviation of the standard deviation

The standard deviation of the standard deviation can be shown to be $\mathbf{e}^{\mathbf{1 0}}$ :

$$
\sigma(\sigma)=\sqrt{\frac{2 \bar{r}+\mathrm{r}}{4^{N}}}
$$

where $N$ is the number of stars counted. It is important to remember that the standard deviation of the variance is not merely the square of the above quantity.

\section{RESULTS}

In these experiments the rate of inactivation of the phage, due to the decay of incorporated ${ }^{32} \mathrm{P}$, the so-called suicide effect, was used as a secondary standard for the determination of the amount of ${ }^{32} \mathrm{P}$ in the phage population. The efficiency of killing has been measured in other laboratories, ${ }^{3}, 11$ and also confirmed by us, and it has been found that the inactivation of phage proceeds exponentially with the number of ${ }^{32} \mathrm{P}$ atoms which have decayed according to the following expression:

$$
\ln P / P_{0}=-\left(\mathrm{I}-\mathrm{e}^{-\lambda t}\right) \alpha N_{0}
$$

where $P / P_{0}$ is the fraction of the phage population which remains viable, $\lambda$ is equal to the fraction of ${ }^{32} \mathrm{P}$ decays per unit time, $N_{0}$ is the average number of ${ }^{32} \mathrm{P}$ atoms per phage particle at time zero, and $\alpha$ is the so-called efficiency of killing. For determination of the ${ }^{32} \mathrm{P}$ content of the phage stocks used in the emulsion experiment, inactivation rate was determined by daily assays of the diluted phage stock, and the inactivation rate is reported in terms of $a N_{0}$. The phage are put on the emulsion either after purification by differential centrifugation and DNase and RNase treatment, or, after further purification by adsorption to sensitive bacteria and separation of these infected bacteria by means of centrifugation. In either case, the same results are obtained and Fig. 4 shows several calibration curves which were obtained by measuring the number of rays per star as a function of the fraction of ${ }^{32} \mathrm{P}$ atoms which have decayed during the exposure time. For each experiment the inactivation by suicide was measured and the results are summarized in Table I.

TABLE I

\begin{tabular}{ccc}
\hline $\begin{array}{c}\text { Star counting } \\
N_{0}\end{array}$ & \multicolumn{1}{c}{$\begin{array}{c}\text { Suicide } \\
\alpha N_{0}\end{array}$} & $\begin{array}{c}\text { Eficiency of killing } \\
a\end{array}$ \\
\hline & & \\
$25.3 \pm 0.6$ & $2.88 \pm 0.14$ & $0.114 \pm 0.008$ \\
$44.3 \pm 1.8$ & $4.65 \pm 0.14$ & $0.105 \pm 0.007$ \\
$92 \pm 9$ & 10.5 \pm 0.4 & $0.11 \pm 0.02$ \\
\hline
\end{tabular}

The fact that the observed number of tracks per star increases linearly with the quantity $\mathrm{I}-\mathrm{e}^{-\lambda t}$ in Fig. 4, indicates that there is no significant fading of the latent images over the time of storage which, for the case of the lowest level of labeling shown, is about 3 weeks. The efficiency of killing factor $\alpha$ determined by combining suicide measurements and star counting is $0 . \mathrm{Ir} \pm 0.0 \mathrm{r}$. This is in good agreement with the References p. 465 . 
value of 0.10 previously reported ${ }^{3}, 11$. This means that it is not possible to demonstrate any inefficiency of the emulsion in detecting $\beta$ disintegrations; the detection efficiency of the method is probably no less than $85 \%$.

\section{Measurement of total radioactivity by a Geiger counter and in the emulsion}

The Geiger counter was standardized against a ${ }^{32} \mathrm{P}$ source supplied by the National Bureau of Standards, and found to have an efficiency of $17 \%$. Knowing this, it is possible to relate the counting rate with the number of ${ }^{32} \mathrm{P}$ atoms $/ \mathrm{ml}$ in the sample.

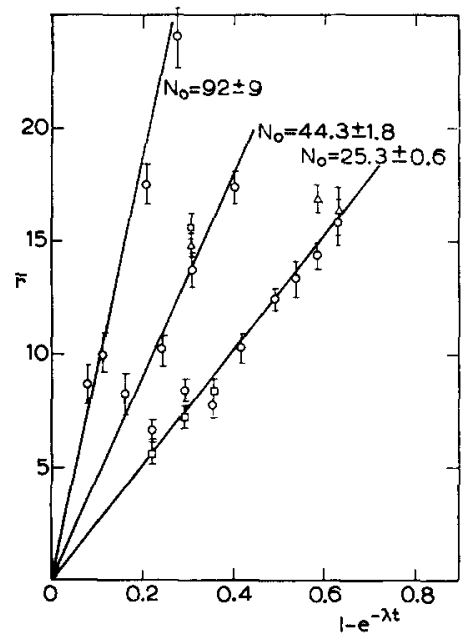

Fig 4.

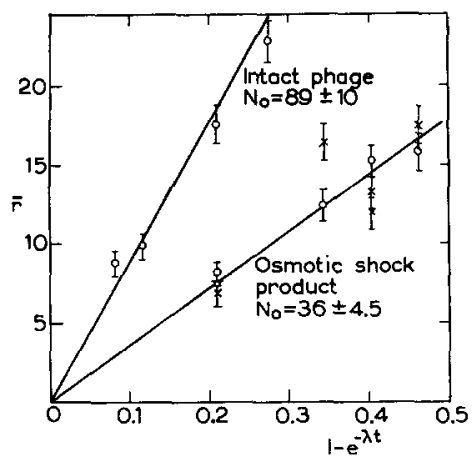

Fig. 6
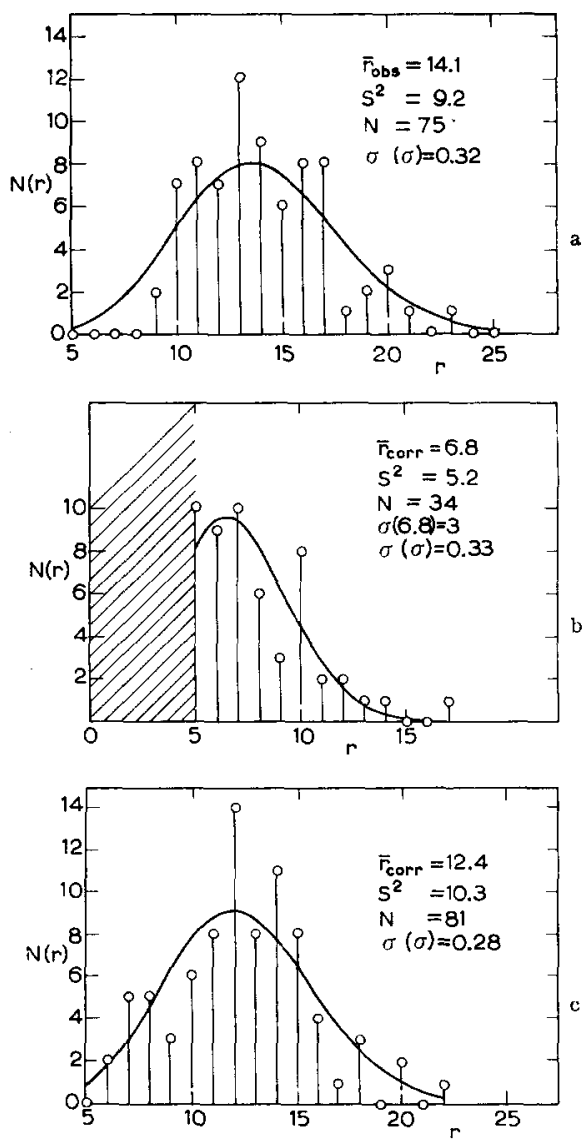

Fig. 5

Fig. 4. Increase of average number of rays per star as a function of the fraction of ${ }^{32} \mathrm{P}$ atoms decayed. The standard deviation of each measurement in indicated by the flags. Three experiments are shown in which the average number of ${ }^{32} \mathbf{P}$ atoms per phage particle is 92,44 , and 25 , respectively.

Fig.5. (a) Histogram of star population resulting from intact $\mathrm{T} 2$ phage. (b) and (c) Histogram of star population of $\mathrm{T}_{2}$ shocked material. The fraction of ${ }^{32} \mathrm{P}$ atoms decayed in (b) is 0.210 , in (c) 0.345 . The data are compatible with Poisson distributions in all three cases.

Fig. 6. The average number of rays per star for intact $\mathrm{T}_{2}$ phage particles and osmotic shock product, both of which were imbedded at the same time. The osmotic shock fragment is shown to have only $40 \%$ of the phosphorus of the intact phage, and this $40 \%$ is in a single uniform piece. The points marked with crosses show the results of osmotically shocking the labeled phage in the presence of a large excess of cold carrier phage, the thought being that a large excess of cold DNA might prevent possible aggregation of two labeled DNA molecules. This had no effect.

References $p .46 .5$. 
When the radioactive phage is adsorbed to sensitive bacteria and the infected bacteria are purified by centrifugation, almost all of the radioactivity in the solution is likely to be in phage which have been adsorbed, and hence in star-forming particles. Since it is possible to determine the total number of stars per $\mathrm{ml}$ by counting the ratio of stars to indicator particles, one can calculate the total number of ${ }^{32} \mathrm{P}$ atoms in the particles of the solution which was mixed with the emulsion.

In Table II the results are given of counting a series of plates which were stored for increasing periods of time before development. The average number of ${ }^{32} \mathrm{P}$ atoms per phage particle, $N_{1}$, was determined as in Fig. 4 and the total number of ${ }^{32} \mathrm{P}$ atoms/ $\mathrm{ml}$ calculated from the number of stars/ml calculated from Geiger counter measure-

TABLE II

$$
N_{1}=\mathrm{I} 26 \pm \text { Io; Counts } / \mathrm{ml} / \mathrm{sec}=\mathrm{I} 55 \pm 9
$$

\begin{tabular}{|c|c|c|c|c|c|}
\hline Star/spheres & Spheres per ml & Dilution & Starsiml ${ }^{*} \times 10^{-7}$ & $\begin{array}{l}\text { Stars } / m l \\
\times N \times{ }^{-\theta}\end{array}$ & $\begin{array}{l}\text { Atoms }{ }^{32} \mathrm{P} / \mathrm{ml} \text { lrom } \\
\text { Geiger counter } \times{ }^{-} \mathrm{O}_{\mathrm{g}}\end{array}$ \\
\hline $\mathrm{I}_{44} / 170$ & $1.75 \times 10^{9}$ & $10^{3}$ & $\mathrm{I} .5^{\circ} \pm 0 . \mathrm{I} 7$ & $1.90 \neq 0.28$ & $\mathrm{I} .63+0.13$ \\
\hline $100 / 131$ & $1.75 \times 10^{9}$ & $\mathrm{IO}^{3}$ & $1.34 \pm 0.18$ & $1.68 \pm 0.26$ & I.63 4 0.1 3 \\
\hline $\mathrm{IO}_{4} / \mathrm{I} 2 \mathrm{I}$ & $1.75 \times 10^{9}$ & $10^{3}$ & $1.50 \pm 0.20$ & $1.90 \pm 0.30$ & $1.63 \pm 0.13$ \\
\hline
\end{tabular}

* The small correction which is indicated by Fig. 3 for $x=5$ is included.

ments is shown in the last column. The agreement is satisfactory in view of the possible systematic errors in the comparison, namely, in the calibration of the Geiger counter, and in the determination of the absolute concentration of indicator particles. It is to be expected that the emulsion should give a slightly lower value for atoms of ${ }^{32} \mathrm{P} / \mathrm{ml}$ since only that radioactivity which is in phage is assayed.

However, the general agreement between the two counting methods as shown in Table II supports the conclusion that the emulsion is highly efficient, and that the number of stars $/ \mathrm{ml}$ can be properly determined by the method of indicator particles.

\section{Uniformity of phosphorus content of intact phage}

A histogram of the star population resulting from a suspension of intact free phage particles is shown in Fig. 5(a). The data are consistent with the Poisson distribution having a mean of $I_{4}$. This implies that the data are compatible with the assumption that the star-forming centers have a perfectly uniform amount of phosphorus.

\section{Fragments resulting from osmotic shock}

Phage particles were disrupted by an osmotic shock procedure ${ }^{13}$ which consisted of diluting into saturated $\mathrm{NaCl}$, and then rapidly adding 20 volumes of distilled water. This procedure has been shown to render the DNA of the phage sensitive to DNase, and to make possible the separation of protein ghosts consisting of the phage tail and a membrane surrounding the head of the phage. It is likely, therefore, that the osmotic shock procedure liberates the DNA free in solution.

The solution resulting after osmotic shock was further diluted and mixed with the melted emulsion. In Fig. 6 the results of counting $\bar{r}$ as a function of exposure is shown. The intact phage and the shocked material were embedded at the same time. It can be seen that the osmotic shock procedure reduces the number of ${ }^{32} \mathrm{P}$ atoms per starforming center by $40 \pm 6 \%$. 


\section{Number of fragments per phage particle}

The polystyrene latex spheres were used as indicator particles in order to determine the number of fragments resulting from the osmotic shock of the phage. The spheres were mixed with the phage suspension before shock, and both the osmotically shocked and the material which was diluted slowly without osmotic shock were embedded in the emulsion. The results are shown in Table III.

TABLE III

\begin{tabular}{|c|c|c|c|}
\hline & \multicolumn{2}{|c|}{ Starispheres } & \multirow{2}{*}{ Fragmentsiphage particle } \\
\hline & Original phage & Shocked phage & \\
\hline \multirow[t]{2}{*}{$\mathrm{T}_{2}$} & $\begin{array}{c}91 / 94 \\
116 / 122\end{array}$ & $\begin{array}{c}68 / 74 \\
104 / 106\end{array}$ & $0.99 \pm 0.13$ \\
\hline & $100 / 102$ & & \\
\hline \multirow[t]{2}{*}{$T_{4}$} & $100 / 120$ & $105 / 115$ & $1.02 \pm 0.13$ \\
\hline & I I $6 / 127$ & $101 / 116$ & \\
\hline
\end{tabular}

Each entry in this table records the results of counting the number of stars and indicator particles in an arbitrary volume of emulsion on different plates which were developed at different times. The fact that the ratio of stars to spheres is the same for both the intact phage and shocked material leads at once to the conclusion that there is one large fragment per phage particle. Since this fragment comprises $40 \%$ of the phosphorus in the phage, the remaining $60 \%$ must be in pieces too small to form stars at this level of labeling.

\section{Uniformity of fragments resulting from osmotic shock}

Fig. 5(b) and (c) are histograms of the star population resulting when the osmotically shocked material from the phage $\mathrm{T} 2$ was exposed for two different lengths of time. The data are consistent with a Poisson distribution as judged by the $\chi^{2}$ test and are therefore compatible with the concept of a perfectly uniform piece. If a small amount of non-uniformity exists, equation 8(a) allows one to estimate the degree of polydispersity which would go unnoticed by these observations. If the true molecular weight distribution had a standard deviation of about $\mathrm{I} 6 \%$, Figs. 5(b) and (c) would not be expected to be markedly different from their present appearance.

If the decrease in star size after osmotic shock were due to the random fragmentation of a single linear DNA molecule, an average of $\mathrm{I} .5$ to 2 breaks per parent molecule must be postulated to account for the decrease in molecular weight observed ${ }^{12}$. We would expect a fraction of $e^{-1.5}$ to $e^{-2}$ or $22 \%$ to $14 \%$ of the molecules to have suffered no breaks, and therefore give rise to a population of stars which have full size, namely, with $N_{0}$ values corresponding to intact phage. Thus in Fig. 5 (c) we would expect to find II to I 8 stars with approximately 3 I rays. Since these are not observed, this possibility is clearly eliminated. A second argument is based on the increased polydispersity attendant on random degradation. If 1.5 to 2.0 breaks per parent molecule occurred, $t_{w} / t_{n}$ would be about $\mathrm{I} .5$ to $\mathrm{I} .7$; thus from equation 8 the variance in the star population, $\sigma^{2}$, would be about Ioo, in Fig. 5(c). Since the observed variance is about I9 \pm 2 the hypothesis of random fragmentation is untenable. The random association of smaller pieces of DNA would also be expected to lead to a polydisperse References $p .465$. 
system in which $t_{w} / t_{n}$ would be 2.o. This would give from equation $8 \sigma_{r}^{2}=\mathrm{I} 54$. As in the former case, the small observed variance rules out this possibility.

The fact that stars of size 5 or less are not counted makes a small correction to the above figures, but does not change the argument.

\section{Treatment of shocked material with DNase and chymotrypsin}

If the shocked material is treated for Io minutes with DNase (during which time the specific viscosity is reduced to zero) the number of stars decreases by a factor of 400 . The remaining stars are of such size as to correspond to intact phage particles which had not been shocked. The stars from $\mathrm{T}_{2}$ and $\mathrm{T}_{4}$ in the shocked material do not decrease in size or number after two hours digestion in chymotrypsin.

\section{DISCUSSION}

The results reported above show that it is possible to count the $\beta$ disintegrations from single particles in nuclear emulsions. The efficiency has been established within our limits or error, and cannot be shown to differ from $100 \%$ and is probably not lower than $85 \%$. This efficiency remains constant with exposure time up to three weeks, the longest observation made. The counting is certainly more tedious than the use of a Geiger counter; however, the data can be obtained in a reasonable amount of time, and it is estimated that an experienced observer can count between 25 and 50 stars per hour. It is not entirely clear how many other applications this method might have. For any work with bacteria it is certainly possible to determine the labeling in the bacteria as a whole. There are, on the other hand, rather few molecules which could possibly have the size of the piece of DNA observed in these experiments. Therefore, it is to be expected that most other applications of the method will not involve the use of single molecules, but will more likely involve the use of single microorganisms. With bacteria it is probable that many $\beta$-emitting isotopes could be used and a sufficient level of labeling could be obtained with them. The counting would undoubtedly be more difficult if one used an isotope, as, for example, carbon-I4, where the $\beta$ energy is considerably lower than in the case of phosphorus. However, no experiments have been done by us with any isotopes other than phosphorus- 32 .

The observation that the phages $\mathrm{T}_{2}$ and $\mathrm{T}_{4}$ have a piece of DNA of a very high molecular weight which remains intact during osmotic shock, brings up several additional questions which have nothing to do with the detection method itself. The first question is, what is the evidence that this piece of DNA is really free in solution and not simply attached to the ruptured protein membranes. Since this particle has been made sensitive to DNase and is of a unique size, it is likely that the particle is free in solution, but this conclusion is by no means established. It has not been possible to separate the DNA from the protein, after osmotic shock, by centrifugation. However, this could be due to the fact that there is an aggregation of the DNA and the protein if one uses concentrations which are necessary to form a pellet in the centrifuge. In our case, the concentrations involved are less by a factor of $10^{\theta}$ than those necessary for centrifugation.

If we consider the DNA contents of a phage to be made up of two components, a single large piece comprising $40 \%$ of the phosphorus of the virus and smaller pieces representing $60 \%$ of the total phosphorus, it can be shown that there must be six or References $p .46 .5$. 
more of these small pieces (of not too widely varying sizes). Otherwise they would begin to reveal themselves in Fig. 5(c). Most physical methods for molecular weight determination applicable to DNA measure something close to a weight average molecular weight. We therefore want to know what values of weight average molecular weight are compatible with a single large piece of DNA and 6 or more small pieces. It can be shown that $\bar{t}_{w}=\frac{0.60 \phi t_{w}^{s}+(0.40 \phi)^{2}}{\phi}$ where $\bar{t}_{\mathfrak{w}}$ is the weight average degree of polymerization of the entire DNA population; $t_{w}^{s}$ is the corresponding weight average for the small pieces only, and $\phi$ is the total number of phosphorus atoms per phage particle. If $t_{w}^{s}$ becomes very small (very many small pieces) then $\bar{t}_{w}$ approaches but does not fall below o.I $6 \phi$, corresponding to a molecular weight of 19 million. This leads at once to the conclusion that the weight average molecular weight of phage DNA is greater than I million.

Since $t_{w}^{s}$ must be smaller than $\phi / I 0$ in order to escape observation, the upper limit of $\bar{t}_{w}$ compatible with our results is $0.22 \phi$ corresponding to a molecular weight of 27 million. Therefore, any values for the weight average molecular weight between I9 and 27 million are compatible with our results.

The second question which must be raised about this large piece of DNA is whether or not it existed as such in the phage or whether in some way it was formed during the osmotic shock procedure. From the fact that it is unique in size, it seems likely that it existed in some sense inside the bacteriophage. It might, however, have been some part of a larger piece which was broken at a discrete point during osmotic shock.

Probably the most interesting question about this large piece of DNA is that concerning its function. Experiments are under way ${ }^{14,15}$ which seem to imply that the big piece of DNA contains some, if not all, of the genetic markers of the phage. However, we have at the moment no method of establishing clearly the function of the small pieces of DNA. The probability that they transfer their atoms to progeny phage has been studied and they seem to transfer with an efficiency equal to that of atoms in the large piece. However, it has not been possible to establish whether or not they play a significant function in the growth of the bacteriophage.

\section{ACKNOWLEDGEMENTS}

We should like to thank Miss Joyce Molin, Mr. Maurice Barancik, and Mr. Richard HEDE for their assistance during this work. We are grateful to Dr. JOHN VANDERHOFF of Dow Chemical Company for providing the polystyrene indicator particles.

\section{SUMMARY}

A method for measuring the radioactivity of single DNA molecules and virus particles by imbedding them in a sensitive nuclear emulsion is described. The technique is probably roo\% efficient in detecting high energy $\beta$-rays and is many thousandfold more sensitive than conventional Geiger counters because of the effective elimination of background. With this new technique the distribution of ${ }^{32} \mathrm{P}$ atoms in intact phage and in the fragments of phage resulting from osmotic shock has been explored. The results indicate than $\mathrm{T}_{2}$ and $\mathrm{T}_{4}$ phage contain a single phosphorus-containing molecule, resistant to protease, but sensitive to DNase, which could be the chromosome of the virus. 


\title{
REFERENCES
}

1 H. Levi, Exptl. Cell Research, 7 (I954) 44.

2 A. Ficg, F. Gavosto and M. Erra, Exptl. Cell Reseavch, 6 (I954) 69.

3 A. D. Hershey, M. D. Kamen, J. M. Kennedy and H. Gest, J. Gen. Physiol., 34 (I95I) 305.

${ }^{4}$ C. Levinthal, Rend. inst. lombardo sci., 89 (1955) ig2.

5 C. Levinthal, Proc. Natl. Acad. Sci. U.S., 42 (1956) 394.

${ }^{6}$ A. Beiser, Revs. Mod. Phys., 24 (1952) 273.

7 M. H. Adams, Methods in Med. Research, 2 (1950) I.

8 A. D. Hershey, Virology, I (1955) Io8.

9 A. H. Doermann, J. Bacteriol., 55 (1948) 257.

${ }^{10}$ T. C. FRY, Probability, D. van Nostrand Co., New York, 1928.

11 G. S. Stent and C. Fuerst, J. Gen. Physiol., 38 (I955) 44I.

12 C. A. Thomas Jr. and P. Doty, J. Am. Chem. Soc., 78 (I956) I 854.

${ }^{13}$ R. M. Herriot, J. Gen. Physiol., 34 (195I) 76r.

${ }^{14}$ C. Levinthal and C. A. Thomas, JR., in Chemical Basis of Heredity, McCollum-Pratt Inst., Baltimore, 1956 (in press).

15 A. D. Hershey AND E. Burgi, Cold Spring Harbor Symposia Quant. Biol., (1956) (in press).

Received September Ioth, I956

\section{THE X-RAY MOLECULAR WEIGHT OF HEMERYTHRIN FROM PHASCOLOSOMA GOULDI AND SOME NOTES ON THE OXYGENATION REACTION*}

\author{
WARNER E. LOVE ${ }^{\star \star}$ \\ The Eldridge Reeves Johnson Foundation for Medical Physics, University of Pennsylvania, \\ Philadelphia, Pa., and Marine Biological Laboratory, Woods Hole, Mass. (U.S.A.)
}

Hemerythrin is a protein which combines reversibly with oxygen and is found in erythrocyte-like cells. Representatives of three phyla possess it. It is present in the nucleated coelomic cells of all the gephyrean annelids examined ${ }^{\mathbf{1}}$, the priapulids Halicryptus spinulosus and Priapulus caudatus ${ }^{2}$, and the escardine brachiopod Lingula unguis $^{3}$. It is also found in the non-nucleated corpuscles of the polychaete annelid Magelona papillicornis ${ }^{4}$. The European gephyrean, Sipunculus nudus has been used for most of the studies to date. One of the purposes of the present paper is to compare Sipunculus hemerythrin with that from Phascolosoma gouldi, the common gephyrean of Woods Hole.

Improvements in the determination of dissociation curves by spectrophotometry ${ }^{5}$ and in the theory of multiple equilibria $6,7,8$ made it of interest to look into some of the properties of hemerythrin. The available data from the early I930's on Sipunculus hemerythrin indicated the ratio $\mathrm{Fe} / \mathrm{O}_{2}$ to be $3 / \mathrm{r}(\mathrm{mole} / \mathrm{mole})^{1}$, and the molecular

\footnotetext{
${ }^{\star}$ This work was supported by a grant from the National Science Foundation.

** Present address: Institute for Cancer Research, 77 or Burholme Avenue, Fox Chase, Philadelphia II, Pa.
} 